\title{
Characterization of Canine Dendritic Cells in Healthy, Atopic, and Non-allergic Inflamed Skin
}

\author{
Meret Elisabeth Ricklin • Petra Roosje • \\ Artur Summerfield
}

Received: 9 June 2010 / Accepted: 15 July 2010 /Published online: 31 July 2010

(C) Springer Science+Business Media, LLC 2010

\begin{abstract}
Atopic dermatitis in humans and dogs is a chronic relapsing allergic skin disease. Dogs show a spontaneous disease similar to the human counterpart and represent a model to improve our understanding of the immunological mechanisms, the pathogenesis of the disease, and new therapy development. The aim of the study was to determine the frequency and phenotype of dendritic cells (DC) in the epidermis and dermis of healthy, canine atopic dermatitis lesional, and non-allergic inflammatory skin to further validate the model and to obtain insights into the contribution of DC to the pathogenesis of skin diseases in dogs. We first characterized canine skin DC using flow-cytometric analysis of isolated skin DC combined with an immunohistochemical approach. A major population of canine skin dendritic cells was identified as $\mathrm{CD} 1 \mathrm{c}^{+} \mathrm{CD} 11 \mathrm{c}^{+} \mathrm{CD} 14^{-} \mathrm{CD} 80^{+} \mathrm{MHCII}^{+-}$ MAC387 $7^{-}$cells, with dermal DC but not Langerhans cells expressing CD11b. In the epidermis of lesional canine atopic dermatitis and non-allergic inflammatory skin, we found significantly more dendritic cells compared with nonlesional and control skin. Only in canine atopic dermatitis skin did
\end{abstract}

\section{E. Ricklin $(\varangle) \cdot$ P. Roosje}

Division of Clinical Dermatology, Department of Clinical

Veterinary Medicine, Vetsuisse Faculty, University of Bern,

Dermatology Unit, Länggass-Strasse 124,

3001 Bern, Switzerland

e-mail: meret@ricklin.info

P. Roosje

DermFocus, Vetsuisse Faculty, University of Bern,

Bern, Switzerland

A. Summerfield

Institute of Virology and Immunoprophylaxis,

Mittelhaeusern, Switzerland we find a subset of dendritic cells positive for $\operatorname{IgE}$, in the epidermis and the dermis. Under all inflammatory conditions, dermal dendritic cells expressed more CD14 and CD206. MAC $387^{+}$putative macrophages were absent in healthy but present in inflamed skin, in particular during non-allergic diseases. This study permits a phenotypic identification and differentiation of canine skin dendritic cells and has identified markers and changes in dendritic cells and macrophage populations related to allergic and nonallergic inflammatory conditions. Our data suggest the participation of dendritic cells in the pathogenesis of canine atopic dermatitis similar to human atopic dermatitis and further validate the only non-murine spontaneous animal model for this disease.

Keywords Skin · atopic dermatitis · dendritic cells · canine

\section{Introduction}

Atopic dermatitis (AD), also called atopic eczema, is a chronic recurrent allergic skin disease with characteristic clinical features that affects humans and domestic animals [1]. The prevalence in children is between $15 \%$ and $30 \%$ of which around $70 \%$ show spontaneous remission before adolescence. Therefore, in adults, the prevalence is much lower (2-10\%), although atopic children often develop asthma and allergic rhinitis, a disease connection called "atopic March" [2]. Canine atopic dermatitis (cAD) affects approximately $10-15 \%$ of the canine population [3, 4]. Spontaneous remission and the atopic March are very rare in dogs [1].

In most cases, human $\mathrm{AD}$ (hAD) and $\mathrm{CAD}$ are associated with allergen-specific IgE [4] and a dysfunction of the epidermal barrier $[1,2]$. The latter is considered a 
prerequisite for the penetration of high molecular-weight allergens originating from pollens, house dust mite products, microbes, and food [2]. Reduced expression of epidermal filaggrin and increased TEWL, indicating impaired epidermal barrier function, are also features of cAD [5]. A genetic predisposition toward AD was shown in dogs as well [6].

Dogs suffering from AD show very similar lesions in type and distribution to hAD [1]. Increased staphyloccocal colonization, recurrent bacterial, and yeast infections are observed frequently and further contribute to pruritus, the most common clinical sign of AD. Self-trauma can aggravate secondary infections. The body parts typically affected in dogs involve the feet and flexural areas of the limbs, axillae, groin, pinnae, periocular, and perioral areas $[1,2]$. Like in man, there is no specific diagnostic test for diagnosis of cAD. Diagnosis of cAD is based on clinical criteria defined by Willemse [7], Prélaud [7, 8], and recently Favrot [9] for dogs. In addition, relevant differential diagnoses such as scabies, flea-bite hypersensitivity, food adverse reactions, and contact allergies should be ruled out. For both humans and dogs, the histopathological alterations of $\mathrm{AD}$ include a spongiotic dermatitis with a mononuclear infiltrate, composed of $\mathrm{T}$ cells and dendritic cells (DC) $[1,2]$. The inflammatory reaction in hAD is biphasic. The initial acute Th2 response dominated by IL-4, IL-5, and IL-13 is $48 \mathrm{~h}$ after atopy patch test followed by a chronic phase in which Th1 cells secreting IFN- $\gamma$, IL-12, IL-5, and GM-CSF infiltrate the skin lesions [2]. In dogs, only the initial Th2 response is typically found, and in chronic lesions, the expressed cytokine pattern can be variable [1].

DC represent a complex family of cells comprised of multiple subsets with different origin, anatomical localization, and function [10]. Besides their particular ability to act as sentinels of the immune system by sensing invading pathogens and taking up antigens and allergens, they are most effective in antigen presentation and are able to prime naive $\mathrm{T}$ cells [11], as well as regulating the orientation of distinct $\mathrm{T}$ cell responses [10]. During the pathogenesis of $\mathrm{AD}$, it is considered that $\mathrm{DC}$ not only take up the allergen but also stimulate key events promoting the clinical manifestation [12]. Understanding these events requires the consideration of DC heterogeneity in the skin. In healthy skin, there are three cutaneous DC populations forming a continuous network. These are the epidermal Langerhans cells (LC) containing Birbeck granules (human LC are characterized by $\mathrm{CD}^{+}{ }^{+} \mathrm{CD} 11 \mathrm{~b}-\mathrm{MHCII}^{+}, \mathrm{CD} 207^{+}$) [12], the resident dermal DC in man composed of three major subsets a $\mathrm{CD} 1 \mathrm{a}^{\text {high }} \mathrm{CD} 44^{+} \mathrm{CD} 14^{-} \mathrm{CD} 16^{-} \mathrm{CD} 206^{\text {high }} \mathrm{CD} 207^{-} \mathrm{CD} 209^{+}$, a $\mathrm{CD} 1{ }^{\text {low }} \mathrm{CD} 4^{+} \mathrm{CD} 14^{+} \mathrm{CD} 16^{-} \mathrm{CD} 206^{\text {low }} \mathrm{CD} 207^{-} \mathrm{CD} 209^{+}$population [12], and plasmacytoid DC [13]. During inflammation, there is an additional subset of dermal "inflammatory"
DC in the epidermis and dermis called inflammatory epidermal DC (IDEC) based on their first description in the epidermis. Their phenotype is $\mathrm{CD} \mathrm{a}^{+} \mathrm{Fc} \varepsilon \mathrm{RI}^{+} \mathrm{F} c \varepsilon \mathrm{RII}{ }^{+}$ $\mathrm{IgE}^{+} \mathrm{CD} 206^{+} \mathrm{CD} 11 \mathrm{~b}^{+} \mathrm{CD} 1 \mathrm{~b}^{+} \mathrm{MHCII}^{+} \mathrm{CD} 207^{-}$, and they lack Birbeck granules [13]. Mouse models indicate that these DC are of monocytic origin and may carry antigens to the draining lymph nodes [11]. Monocytes may also replenish LC under inflammatory conditions [14]. In hAD, a significant infiltration of IDEC can be found [15]. All epidermal and dermal $\mathrm{DC}$ populations in $\mathrm{AD}$ express FceRI, which is required to provoke eczematous skin lesions $[16,17]$. On ligation of FceRI by IgE, LC produce IL-16 and IL-13, which recruit monocytes, IDEC, and $\mathrm{CD} 4+\mathrm{T}$ cells to the skin. This in turn induces the production of IgE [18] contributing to the Th2 response. IDEC are considered responsible for the switch to the Th1 response in chronic hAD by producing IL-12 and IL-18 and releasing proinflammatory cytokines $[2,19]$. In humans, $\mathrm{AD}$ skin is practically devoid of $\mathrm{pDC}$.

Due to the spontaneous occurrence and the similarities between cAD with hAD, the dog is a good and unique model to study disease pathogenesis and novel therapeutics [1]. The disadvantage of mouse models are the artificial induction required and the important differences in pathogenesis as a consequence of this $[4,20]$. Another interesting aspect of the spontaneous cAD model is that dogs and people live in the same physical environment, which is an important factor in disease pathogenesis. The DC system of the canine skin has not yet been characterized with exception of one study on cAD describing an increase of $\mathrm{CD}^{+}{ }^{+} \mathrm{IgE}^{+}$cells in the epidermis and dermis [21].

Based on this, the aim of the present study was to characterize the canine skin DC system in more detail in healthy, atopic skin, and selected non-allergic inflammatory skin disease in order to further develop the canine model, and to demonstrate the involvement of DC in the pathogenesis of $\mathrm{cAD}$.

\section{Materials and Methods}

Animals

Control skin biopsies were taken from healthy laboratory Beagles (which served as control dogs in toxicological studies of Novartis Animal Health, Basel, Switzerland) or from privately owned dogs euthanized for reasons not related to this study. Skin biopsies from privately owned dogs suffering of $\mathrm{cAD}$ or non-allergic inflammatory skin diseases (NAI) were also obtained. All biopsies were collected with the owner's written consent. All procedures were approved by the local animal welfare authorities. 
Isolation and Analysis of Skin Dendritic Cells

Skin DC were isolated as published previously [22]. Briefly, an area of skin approximately $8 \times 8 \mathrm{~cm}$ was clipped and collected immediately after euthanasia, and kept on ice in RPMI 1640 (Invitrogen, Lubio Science, Luzern, Switzerland) supplemented with $2 \mathrm{mM}$ L-glutamine (Sigma-Aldrich, Buchs, Switzerland), 1\% streptomycin and penicillin (Sigma), 10\% heat-inactivated FBS (Bioclot GmbH, Hollerbach, Germany), and $1 \%$ fungizone (Sigma) up to $3 \mathrm{~h}$ until processing. The skin was cleared of subcutaneous fat, cut into strips of 1$2 \mathrm{~mm}$, and incubated with $2.4 \mathrm{U} / \mathrm{ml}$ Dispase II (Roche Diagnostics $\mathrm{GmbH}$, Mannheim, Germany) at $37^{\circ} \mathrm{C}$ for $90 \mathrm{~min}$. Epidermis and dermis were then separated with two strong forceps and washed separately twice with PBS at $4^{\circ} \mathrm{C}$. To allow DC migration, the samples were incubated at $37^{\circ} \mathrm{C}$ for $48 \mathrm{~h}$ in medium as described above. In addition, it was supplemented with 1\% Hepes (Invitrogen) and $40 \mathrm{ng} / \mathrm{ml}$ human recombinant GM-CSF (kindly donated by Novartis Pharma, Vienna, Austria) to enhance survival of DC. Migrated cells were harvested by filtering the media through a $70 \mu \mathrm{m}$ Cell Strainer (BD Biosciences, San Jose, CA, USA) to obtain a single cell suspension. Cells were counted and double stained with a rat anti-canine MHC class II antibody (Serotec, Oxford, UK) in combination with a panel of different second monoclonal antibodies (mAb) against $\mathrm{CD} 1 \mathrm{c}$ (CA13.9H11), CD11b (CA163E10), CD11c (CAH.6A1), CD45 (CA4.ID3), CD80 (CA24.5D4), and CD86 (CA24.5E4) (all kindly obtained from Dr. Peter Moore; University of Davis, CA, USA); against IgE (D9) (kindly donated by Dr. D. DeBoer; University of Wisconsin, Madison, WS, USA); against CD4 (CA13.1E4), CD8 (CA9. JD3), CD21 (CA2.1D6), and human CD40 (LOB7/6) (all obtained from Serotec); against CD206 (3.29B1.10) (from Beckman Coulter; Roissy, France); against CD14 (CAM36A) (from VMRD; Pullman, WA, USA); and against MAC387 (from Dako; Baar, Switzerland). The mAb binding was revealed using anti-mouse or anti-rat isotype specific fluorescein isothiocyanate (FITC) or phycoerythrin conjugated goat $F(a b ') 2$ Ig (Southern Biotech, Birmingham, USA). Fluorescence intensities were quantified using a LSRII or FACScan flow cytometer (BD Biosciences), and data were processed using the FlowJo software (Tree Star, Ashland, OR, USA).

\section{Skin Biopsies}

We collected lesional (groin area) and nonlesional (dorsal flank) skin samples with 6-mm biopsy punches (Provet, Lyssach, Switzerland) of 10 privately owned dogs suffering from atopic dermatitis. AD was diagnosed by applying the clinical criteria of Willemse and Prélaud [7,8] and after exclusion of differential diagnoses by intensive flea control and an unsuccessful elimination diet for 8 weeks using a commercial hydrolyzed protein diet, followed by a provocation test and intradermal skin test (IDT), as well as serum allergen-specific IgE determination. All dogs showed high allergen-specific IgE in serum and were tested positive for house dust or forage mites in the IDT. Before taking biopsies, skin was cleared of secondary infections by appropriate antimicrobial treatment. For comparison, also biopsies of lesional skin from dogs suffering from nonallergic inflammatory skin diseases were taken (five cases of pemphigus folliaceus, three cases of leishmaniasis, and two cases of acral lick dermatitis). Of healthy controls, skin biopsies were taken as well as from the groin area and the dorsal flank. Skin samples were frozen with OCT (Medite, Nunningen, Switzerland) in isopentane (Sigma) cooled in liquid nitrogen and then stored at $-80^{\circ} \mathrm{C}$ until processing. Cryosections of $5 \mu \mathrm{m}$ thickness were cut with a cryotome (HM560 MV, Microm International GmbH, Walldorf, Germany), mounted on Superfrost Plus slides (Medite), dried, and stored at $-20^{\circ} \mathrm{C}$ until staining and analysis. For each dog, the skin sections were stained by immunohistochemistry with monoclonal antibodies against CD1c, CD11b, CD11c, MHCII, CD206, CD14, IgE, and MAC387 using the following procedure. Slides were thawed, fixed in acetone (Grogg Chemia AG, Stettlen, Switzerland) with $0.5 \% \mathrm{H}_{2} \mathrm{O}_{2}$ (Merck, Darmstadt, Germany) to block endogenous peroxidase, and rinsed with PBS before blocking with 5\% normal goat serum (Dako) and application of the primary antibody. For detection, the kit K5003 (Dako) was used according to the manufacturer's instructions. Slides were then counterstained with hematoxylin, washed with PBS, and covered with Aquatex (Merck). All slides were analyzed using a Zeiss Axiolab microscope (Carl Zeiss AG, Feldbach, Switzerland) with a crossline graticule (Carl Zeiss). Positive cells in the epidermis were counted along an area of $3 \times 300 \mu \mathrm{m}$ of the basement membrane and five fields of $100 \mu \mathrm{m}^{2}$ of the interfollicular areas in the upper dermis.

For double immunofluorescence detection of MHCII combined with CD1c, CD11b, CD11c, CD14, CD206, IgE, and MAC387, the mAbs were added simultaneously followed by the secondary antibodies conjugated with anti-mouse Ig Alexa fluor 594 (Invitrogen) and anti-rat IgFITC (Southern Biotech), respectively. Nuclei were counterstained with Hoechst 33258 (Sigma) before covering slides with fluorescent mounting medium (Dako). Slides were analyzed and photographed with a Nikon Eclipse 80i microscope (Nikon AG, Egg, Switzerland) using the Open lab 5 software (Improvision, Perkin Elmer, Waltham, MA, USA) and a Retiga 2000R camera (Qimaging, Surrey, Canada).

Using the NCSS 2004 software (NCSS, Kaysville, Utah, USA), a one-way ANOVA Kruskal-Wallis multiple comparison $Z$ value test was calculated for group comparison 
and a one-sample $T$ test used for comparison of nonlesional cAD skin to normal dorsal skin.

\section{Results}

Isolation and Phenotypic Characterization of Putative Canine Skin DC

From the epidermis and dermis of normal control dogs, we were able to isolate a distinct population of cells double positive for MHCII together with CD1, CD11c, and CD80 (Fig 1a, b). The frequency of these cells varied between $2 \%$ and $12 \%$ for the epidermis and $2 \%$ and $9 \%$ for the dermis in healthy skin of different animals $(n=8)$. CD11b and CD86 expression was more variable with low expression in the dermis and often absence in the epidermis (data for CD11b not shown). In normal skin, the putative DC did not express CD40, CD14, and $\operatorname{IgE}$ (Fig 1a, b) and also lacked the lymphocyte markers CD4, CD5, CD8, CD21, and CD45 (data not shown). Light microscopic analyses confirmed the presence of cells with DC morphology in these cell preparations (Fig. 1c).

\section{In Situ Characterization of Canine Skin DC}

To confirm the phenotypic characteristics of putative canine skin DC, biopsies of normal dog skin were analyzed by immunohistochemistry (Fig. 2). In the dermis and epidermis,
Fig. 1 Phenotype and morphology of isolated canine skin DC.

The contour plots show double staining of cells isolated from skin explant cultures with expression of MHCII together with CD1c, CD11c, CD14, CD40, CD80, CD86, CD206, and IgE. Putative DC were identified as MHCII expressing cells which have migrated out of the epidermis (a) and the dermis (b). The regions show the positive populations, and the numbers in the upper right corner indicate the percentage of the positive cells within the gate. In c, a phase contrast photomicrograph of a putative isolated DC (in the upper left corner) is shown $(\times 100$ magnification). The data are representative for five different healthy animals
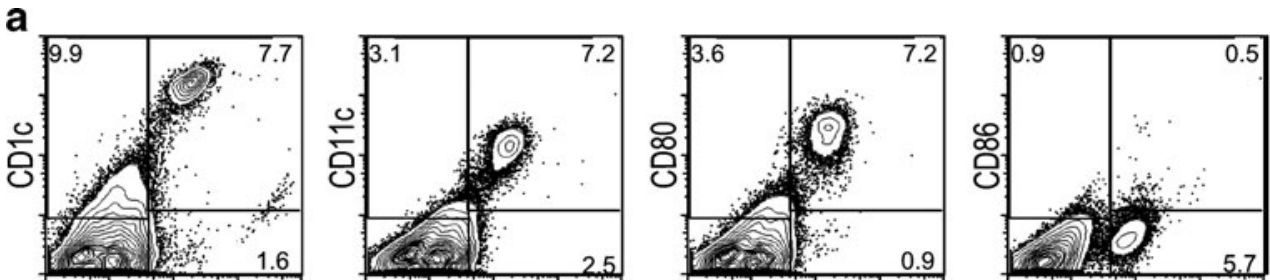

$\mathrm{MHCll}$
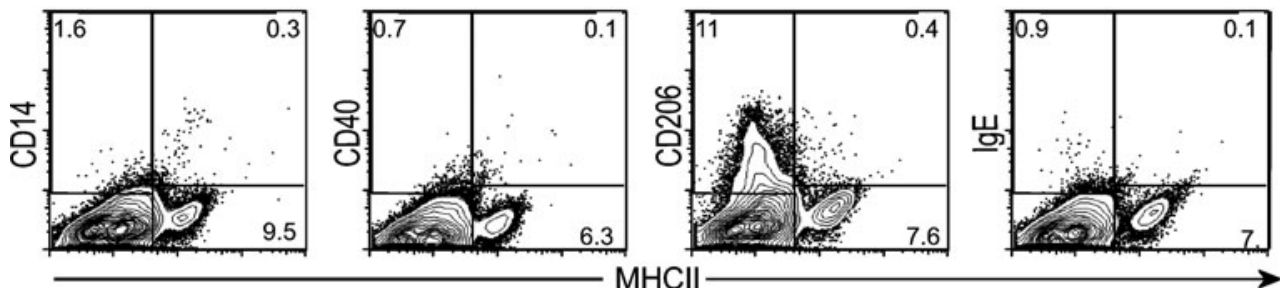

b
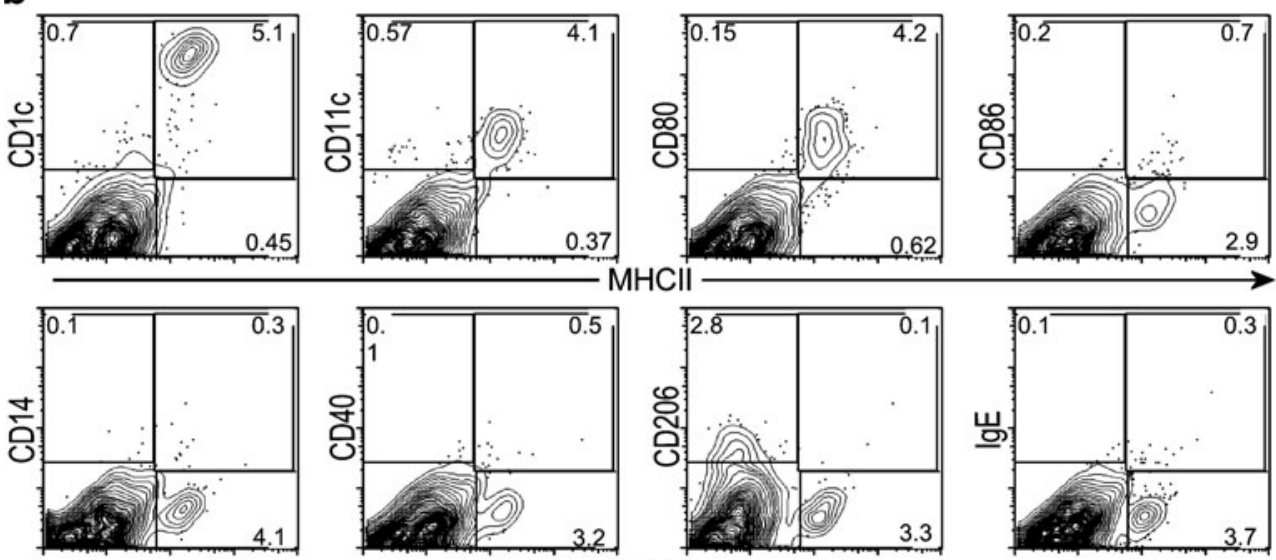

$\mathrm{MHCll}$

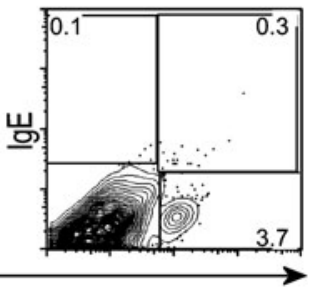

C

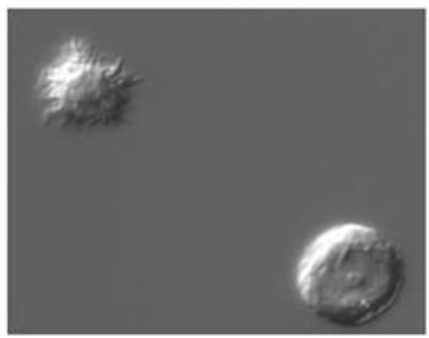


Fig. 2 Phenotype and localization of canine skin DC in situ. Photomicrographs of cryoslides of normal dog skin double stained for MHCII (green) in combination with $\mathrm{CD} 1 \mathrm{c}$, CD11b, and CD11c (all in red). The left and right pictures are from the identical skin area to determine co-expression of the marker. The data are representative for 10 different healthy animals. The dotted line indicates the localization of the basal membrane. Scale bar indicates $20 \mu \mathrm{m}$

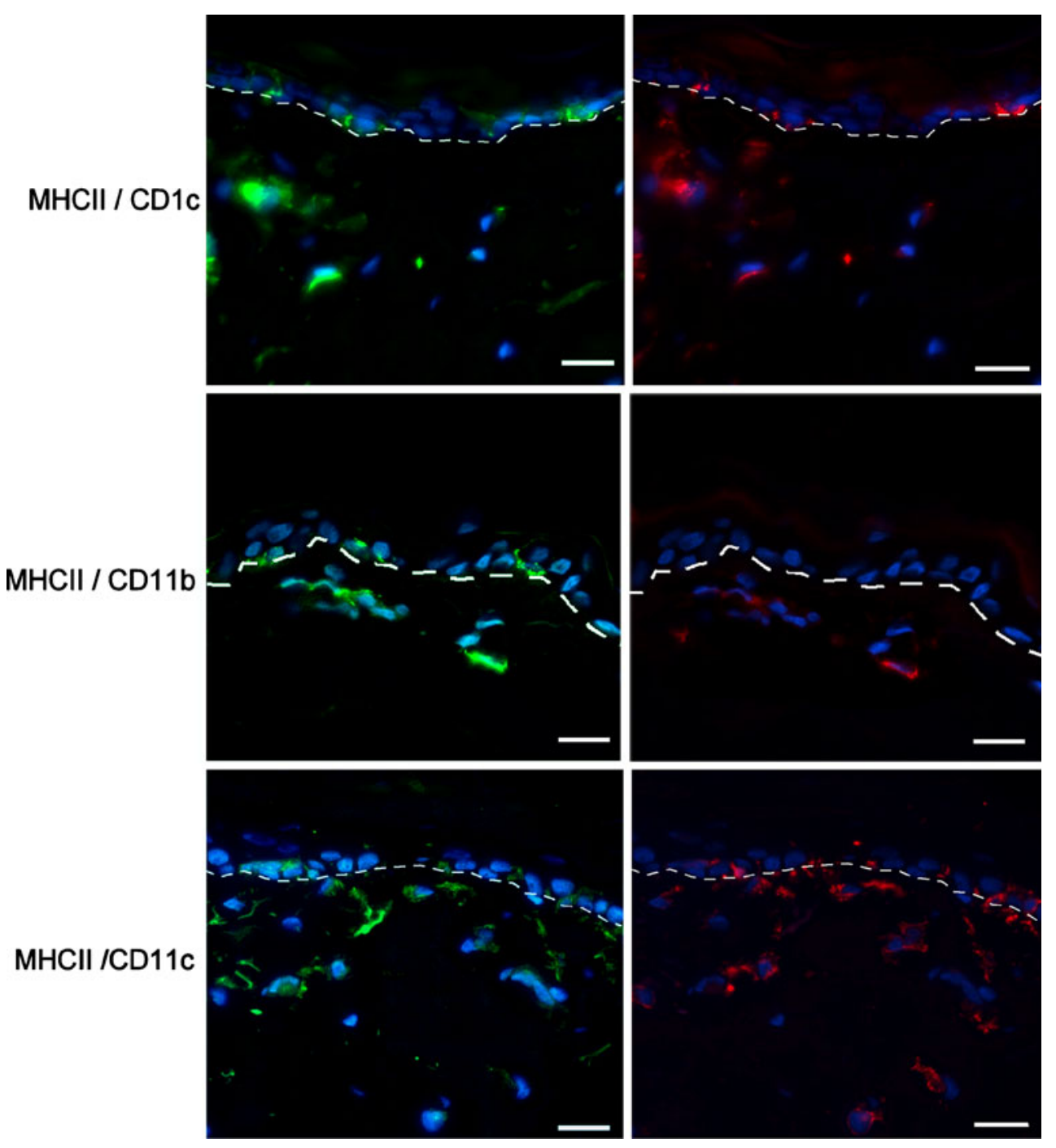

all $\mathrm{MHCII}^{+}$cells had a DC morphology and co-expressed $\mathrm{CD} 1 \mathrm{c}$ and $\mathrm{CD} 11 \mathrm{c}$. Using this technique, CD11b was restricted to the dermis and only found on $\mathrm{MHCII}^{+}$cells with DC morphology. As with flow cytometry, some $\mathrm{MHCII}^{+}$cells in the dermis did not express CD11b.

Based on the morphology, the localization, and the phenotype resembling that of other mammals, we classified the major population of canine skin $\mathrm{DC}$ as $\mathrm{CD}^{+}{ }^{+} \mathrm{CD} 11 \mathrm{c}^{+} \mathrm{MHCII}{ }^{+} \mathrm{CD} 80^{+}$ cells. Because of this analogy to other species, DC present in the epidermis were termed LC.

Frequency, Phenotype, and Localization of DC in Inflammatory Canine Skin

With this information, our next aim was to characterize DC in inflammatory skin. To this end, we compared ventral and dorsal control skin with lesional skin of dogs suffering from cAD or NAI (Fig. 3). Lesional skin showed a mild to severe acanthosis and in the epidermis mild spongiosis with a mononuclear infiltrate, as known in the published literature
[23]. In inflammatory skin diseases (cAD and NAI), we observed an increase of $\mathrm{CD} 1 \mathrm{c}^{+} \mathrm{CD} 11 \mathrm{c}^{+} \mathrm{MHCII}^{+} \mathrm{DC}$ in the epidermis by at least a factor 2 compared with normal skin (Figs. 4a and 5) and a 2-4-fold increase of $\mathrm{CD} 1 \mathrm{c}^{+} \mathrm{CD} 11 \mathrm{c}^{+} \mathrm{MHCII}^{+}$cells in the dermis (Figs. $4 \mathrm{~b}$ and 5 ). Under inflammatory conditions as well as in healthy skin, $\mathrm{CD} 11 \mathrm{~b}$ expression was restricted to the dermis and not found in the epidermis. Despite this general increase of DC, the epidermis and dermis of $\mathrm{cAD}$, but not the NAI skin, showed an increase of $\operatorname{IgE}^{+}$cells. In contrast, the increased number of cells expressing CD206 or CD14 was restricted to the dermis and found in both $\mathrm{cAD}$ and NAI skin. Within the NAI group, this infiltrate was similar in the PF cases $(n=5)$, the leishmaniasis cases $(n=3)$, and also the cases with lick dermatitis $(n=2)$. The appearance of cells expressing the putative macrophage marker MAC387 was restricted to the dermis and more prominent in NAI skin.

With respect to the expression of the above markers, nonlesional dorsal skin biopsies of $\mathrm{cAD}$ dogs were comparable with normal dorsal skin of control dogs, but 

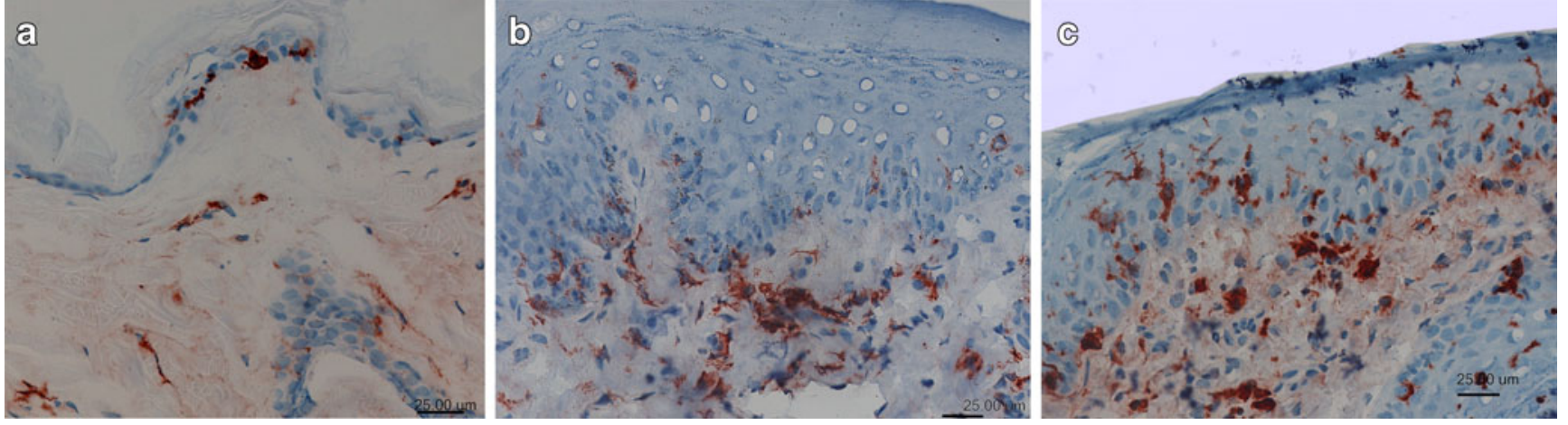

Fig. 3 DC frequency and distribution in healthy, atopic, and nonallergic inflamed canine skin. Photomicrographs of cryoslides stained with CD1c of skin biopsies of nonlesional (a) and lesional (b) atopic skin, and NAI skin (c). Data are representative for 10 different animals for each group. Scale bars indicate $25 \mu \mathrm{m}$ differed significantly to lesional skin with $p$ values of $<0.001$ (Fig. 4c, d).

As demonstrated in Fig. 4, in normal skin, LC were located in the stratum basale, and dermal DC mostly just below the dermo-epidermal junction; in lesional skin, LC were scattered through the acanthotic epidermis (only in NAI conditions) and in the upper dermis. Dendritic cells in the epidermis and dermis co-expressed MHCII and CD1c (Fig. 4a, the plots in the first two rows are from the same areas). Based on this observation, as well as the fact that all DC co-expressed MHCII (Figs. 1 and 2) and that the frequency of $\mathrm{MHCII}^{+}$cells was similar to that of $\mathrm{CD} 1 \mathrm{c}$ and $\mathrm{CD} 11 \mathrm{c}$ in inflammatory conditions, we employed double staining using MHCII together with CD206, CD14, IgE, and MAC387 to investigate the relationship of cells expressing these markers to the DC lineage (Fig. 5b). The results showed that in all $\mathrm{CD} 206^{+}$cells, only a subset of $\mathrm{CD} 14^{+}$and $\mathrm{IgE}^{+}$cells, and no MAC $387^{+}$ cells, co-expressed MHCII. This indicates that during inflammatory conditions, another subset of putative DC infiltrates with an $\mathrm{MHCII}^{+} \mathrm{CD} 206^{+} \mathrm{CD} 14^{+}$phenotype as well as putative macrophages characterized by MAC387 expression.

\section{Discussion}

The dog is genetically quite close to humans, shares the same environment, and suffers similar spontaneously occurring diseases. Therefore, it could be a particular
Fig. 4 Frequency of cells expressing DC markers in healthy, atopic, and non-allergic inflamed canine skin. Cells expressing the indicated markers were counted in immunochemically labeled epidermis (a) and dermis (b) obtained from healthy dogs (light gray bars) as well as from inflammatory skin of animals diagnosed as cAD (black bars) or NAI (dark gray bars). The lower graphs show normal dorsal skin of healthy dogs (light gray) compared with nonlesional cAD skin (black) for the epidermis (c) and dermis (d). The bars show the mean of 10 different animals with standard deviations. 1) indicates statistically significant differences of inflammatory skin to healthy control dogs. 2) indicates statistically significant differences between cAD and NAI skin
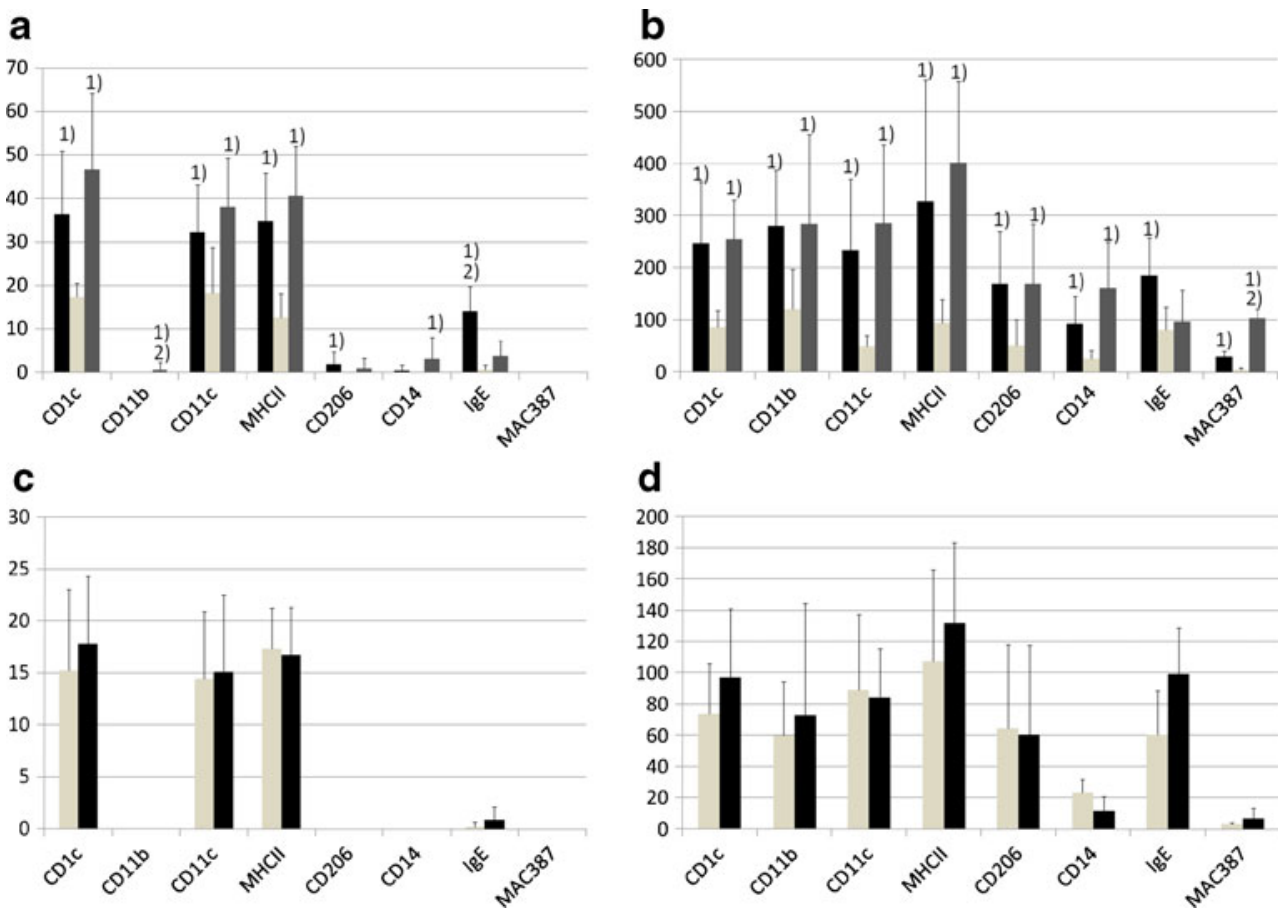
Fig. 5 Phenotype and localization of canine DC in atopic and non-allergic inflamed canine skin. a Photomicrographs of cryoslides of normal dog skin (first column), lesional cAD skin (middle column), and lesional NAI (pemphigus foliaceus) skin double stained for MHCII (green) in combination with CD1c (first two rows), and single stained for CD11c, CD206, CD14, and IgE (all in red) are shown. The data are representative for 10 different animals in each group. In $\mathbf{b}$, $\mathrm{cAD}$ and NAI lesional skin were double stained for MHCII (green) together with CD206, CD14, IgE, and MAC387 (all in red) and the same field is shown. The dotted line indicates the localization of the basal membrane. The data are representative for three different animals in each group. Scale bar indicates $50 \mu \mathrm{m}$ a
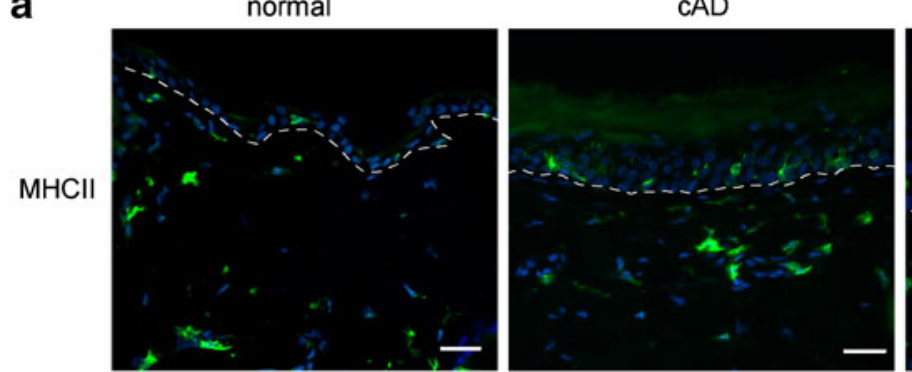

non-allergic inflammatory
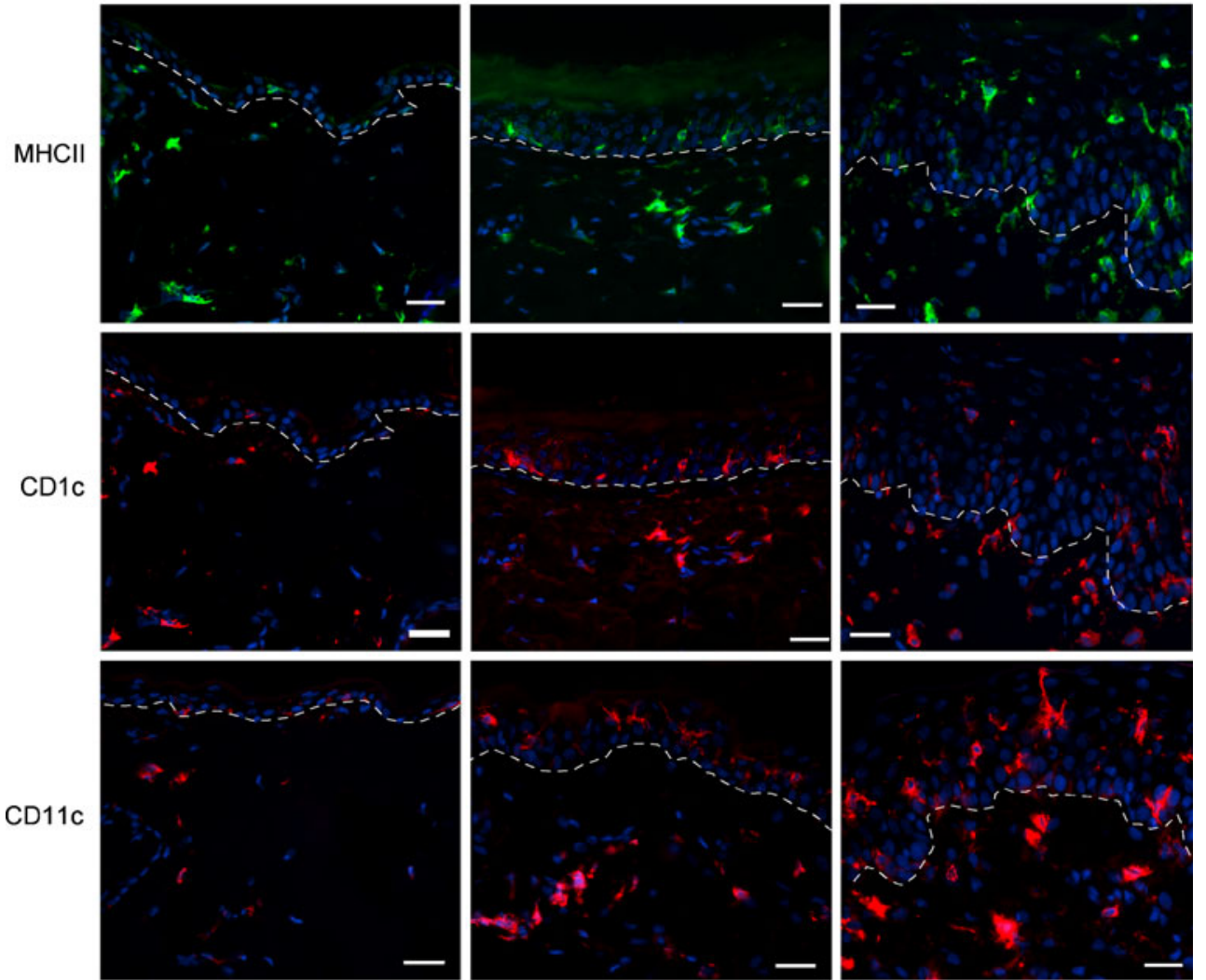

CD11c
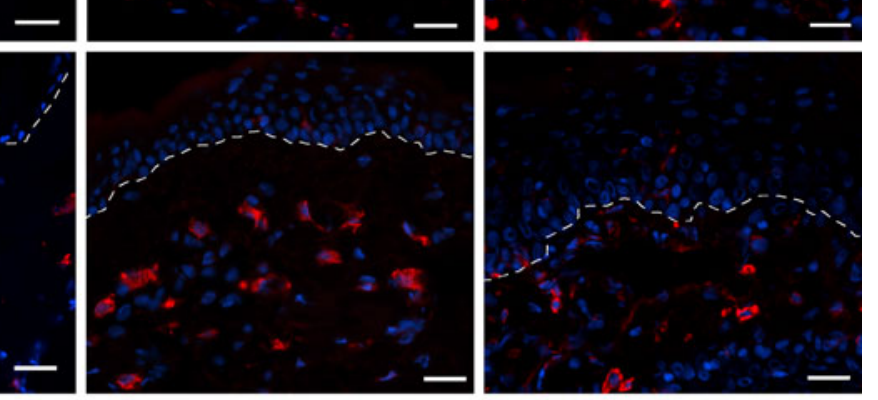

CD206
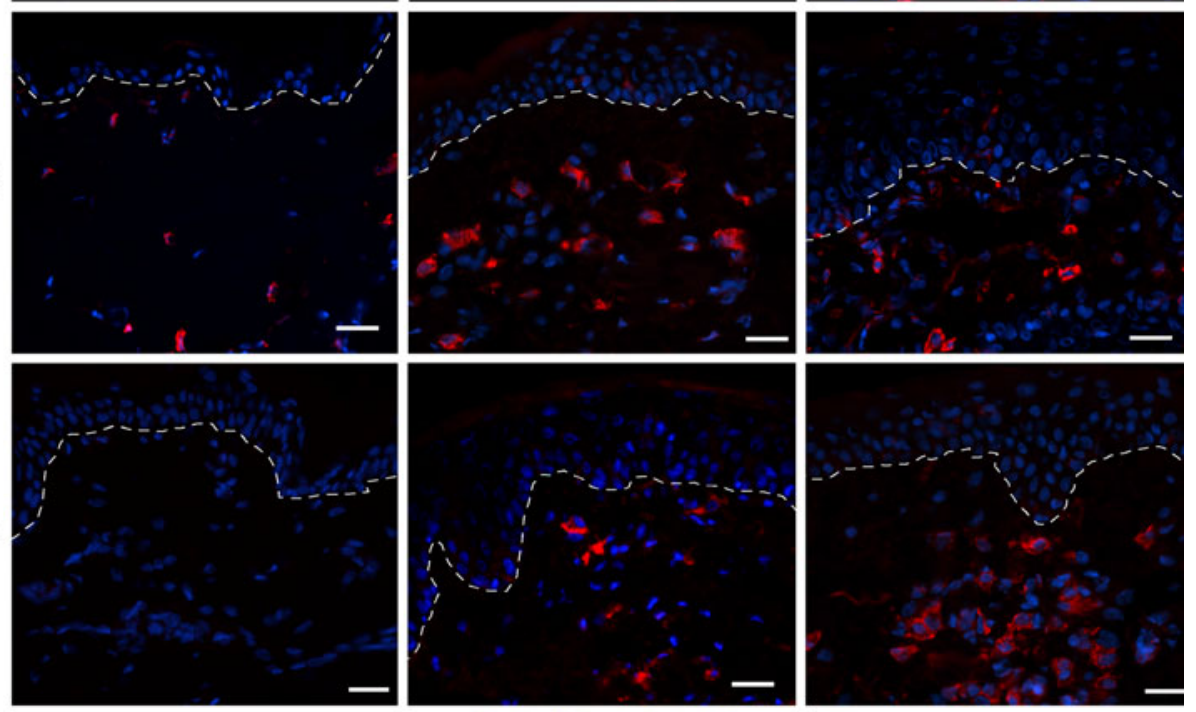

CD14

$\lg \mathrm{E}$
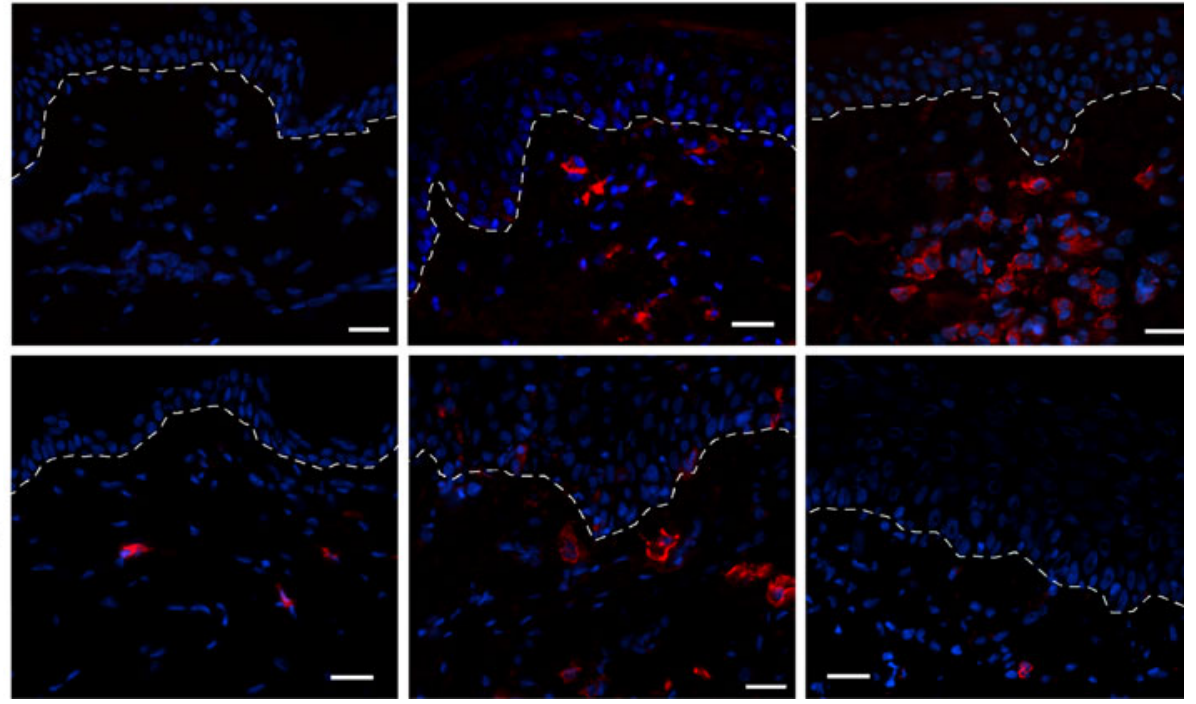


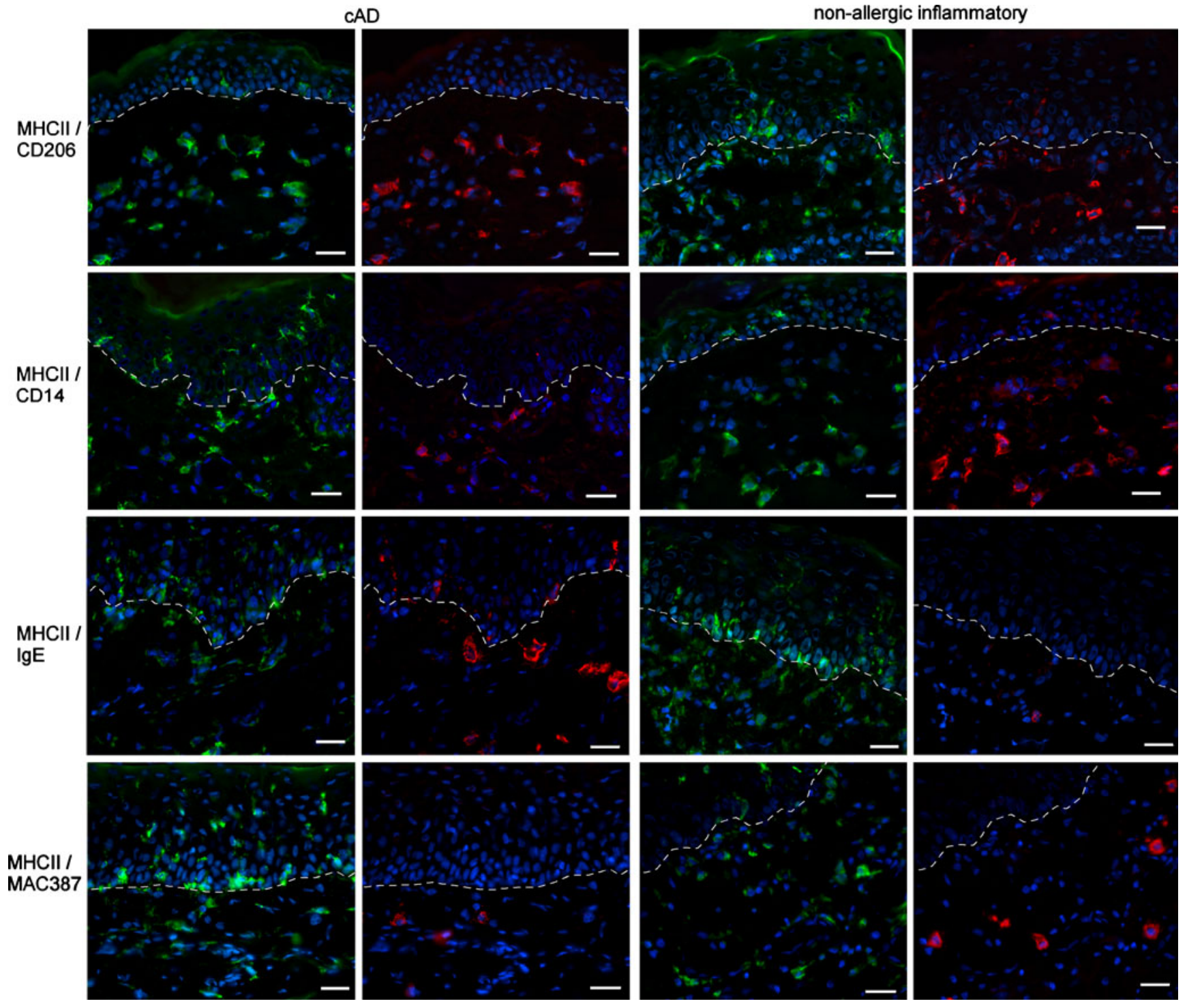

Fig. 5 (continued)

informative animal model for several human diseases such as atopic dermatitis [1], asthma [24], hypothyroidism [25], diabetes mellitus [26], autoimmune hemolytic anemia [27], myopathy [28], and certain infectious diseases [29].

As recently reviewed by Marsella and Girolimo, cAD shows many similarities with the corresponding human disease, in terms of clinical signs, the distribution of the lesions, the pathogenesis, and the immunology of inflammation [1]. Based on the proposed critical role for DC in the pathogenesis of this allergic skin disease in human beings [2], and to provide further support for the canine model for human $\mathrm{AD}$, we performed a systematic characterization of canine skin in healthy dogs compared with animals with cAD or NAI skin diseases focusing on potential DC marker expression.
Our data demonstrate that canine LC express CD1c, CD11c, CD80, and MHCII, and differ from dermal DC by lack of CD11b, although this marker is not expressed on all dermal DC. It is important to note that this differentiation was only evident using the immunohistochemical analyses. The canine LC phenotype is comparable with the human $\mathrm{LC}$, which are $\mathrm{CD} 1 \mathrm{a}^{+} \mathrm{CD} 1 \mathrm{c}^{+} \mathrm{MHCII}^{+}$[13], as well as with porcine $\mathrm{LC}$ defined as $\mathrm{CD}^{+}{ }^{+} \mathrm{MHCII}{ }^{+}$cells in the epidermis [30]. Similarities can also be found with human dermal DC expressing CD1c, CD11c, CD80, CD86, and MHCII [13, 31]. As mentioned above, some canine dermal DC expressed $\mathrm{CD} 11 \mathrm{~b}$ relating to reports of human dermal DC also partially expressing this marker [32]. Our study also demonstrates that the phenotype of the isolated DC resembles that of the in situ stained cells with the exception of CD11b expression. 
One of the main changes in inflammatory skin was an increase in the frequency of $\mathrm{CD} 1 \mathrm{c}^{+} \mathrm{CD} 11 \mathrm{c}^{+} \mathrm{MHCII}^{+} \mathrm{DC}$ in the dermis and epidermis. Typical for $\mathrm{CAD}$ was the appearance of $\operatorname{IgE}^{+} \mathrm{LC}$ and an increase in $\operatorname{IgE}^{+}$dermal DC comparable with several previous studies in dogs $[21$, 33-36] and in human $\mathrm{AD}$, where an FceRI $\mathrm{I}^{+} \mathrm{DC}$ infiltration was observed [13, 15, 37, 38]. In fact, the high expression ratio of $F c \varepsilon R I / F c \varepsilon R I I$ is a diagnostic marker for human $A D$ [39]. The importance of this could be a strong IgEdependent allergen capture and uptake followed by a potent stimulation of T cells [38].

In NAI skin, the infiltrate was comparable with cAD with the difference of missing IgE expression, the higher numbers of infiltrating $\mathrm{CD} 206^{+} \mathrm{CD} 14^{+} \mathrm{MHCII}^{+}$putative $\mathrm{DC}$, as well as the appearance of $\mathrm{MAC} 387^{+}$putative macrophages. The latter were very scarce in normal and $\mathrm{cAD}$ skin but rather frequent in other inflammatory skin diseases, again comparable with human studies [14].

Based on our observation that canine monocytederived DC remain $\mathrm{CD}_{14}^{+}$[40], we propose that the $\mathrm{CD}^{2} 26^{+} \mathrm{CD} 14^{+} \mathrm{MHCII}^{+}$cells represent IDEC, which are a population of monocyte-derived DC that can populate both the epidermis and the dermis under inflammatory conditions [41]. The main difference between IDEC and LC in humans is the lack of Birbeck granules in IDEC [13]. LC in dogs do not have Birbeck granules [42], and we found no reactivity of anti-Langerin antibodies known to react with porcine LC [30]. Therefore, this discriminatory marker is not available, and canine LC can only be defined on the basis of location in healthy skin. Future studies are required to determine the role of these putative IDEC for Th1-mediated skin inflammation as has been proposed for human AD [2]. Other DC subsets such as plasmacytoid DC present in human skin [37] cannot yet be characterized in the dog due to the lack of phenotypic information.

The different phenotypes found in the skin are in part comparable with DC differentiated in vitro [40]. Canine DC in normal skin resemble Flt3-ligand driven bone marrow-derived $\mathrm{DC}$, which are $\mathrm{CD} 1 \mathrm{c}^{+} \mathrm{CD} 11 \mathrm{c}^{+} \mathrm{CD} 11 \mathrm{~b}^{\text {low }} \mathrm{MHCII}^{+} \mathrm{CD} 4^{-} \mathrm{CD} 8^{-}$ and mostly CD206 ${ }^{-}$. The higher levels of CD11b and CD206 on dermal DC in inflammatory skin conditions resemble more the GM-CSF ligand-driven bone-marrow-derived DC.

Taken together, the present study characterized canine LC, dermal DC, and infiltrating inflammatory DC in normal, cAD, and NAI skin adding important information about the canine DC system in healthy and diseased skin. These findings and the identification of discriminatory markers are important for further validation of the dog as a model for human diseases, especially atopic dermatitis and other immunological disorders. Our study confirms that on one side, the cutaneous inflammatory milieu may attract DC precursors and drive their differentiation to different types of inflammatory DC and, on the other hand, that these DC are likely to contribute specifically to disease processes due to their importance in cutaneous homeostasis and their immunological functions [13].

Acknowledgements We are very grateful to Novartis Animal health for sponsoring this study, and acknowledge the help of Vivianne Molitor (University of Bern) for collecting skin biopsies and Catherine Boeglen (Novartis Pharma Basel) for providing healthy control tissue. We appreciate statistical advice of Marcus Doherr.

\section{References}

1. Marsella R, Girolomoni G. Canine models of atopic dermatitis: a useful tool with untapped potential. J Invest Dermatol. 2009;129:2351-7.

2. Bieber T. Atopic dermatitis. N Engl J Med. 2008;358:1483-94.

3. Hillier A, Griffin CE. The ACVD task force on canine atopic dermatitis (I): incidence and prevalence. Vet Immunol Immunopathol. 2001;81:147-51.

4. Marsella R, Olivry T. Animal models of atopic dermatitis. Clin Dermatol. 2003;21:122-33.

5. Marsella R, Samuelson D, Harrington L. Immunohistochemical evaluation of filaggrin polyclonal antibody in atopic and normal beagles. Vet Dermatol. 2009;20:547-54.

6. Shaw SC, Wood JL, Freeman J, Littlewood JD, Hannant D. Estimation of heritability of atopic dermatitis in Labrador and Golden Retrievers. Am J Vet Res. 2004;65:1014-20.

7. Willemse T. Atopic skin disease: a review and a reconsideration of diagnostic criteria. J Small Anim Pract. 1986;27:771-8.

8. Prelaud P, Guaguere E, Alhaidari Z, Faivre N, Heripret D, Gayerie A. Reevaluation of diagnostic criteria of canine atopic dermatitis. Rev Med Vet. 1998;149:1057-64.

9. Favrot C, Steffan J, Seewald W, Picco F. A prospective study on the clinical features of chronic canine atopic dermatitis and its diagnosis. Vet Dermatol. 2010;21:23-31.

10. Liu YJ. Dendritic cell subsets and lineages, and their functions in innate and adaptive immunity. Cell. 2001;106:259-62.

11. Steinman RM, Inaba K, Turley S, Pierre P, Mellman I. Antigen capture, processing, and presentation by dendritic cells: recent cell biological studies. Hum Immunol. 1999;60:562-7.

12. Novak N, Bieber T. The role of dendritic cell subtypes in the pathophysiology of atopic dermatitis. J Am Acad Dermatol. 2005;53:S171-176.

13. Zaba LC, Krueger JG, Lowes MA. Resident and "inflammatory" dendritic cells in human skin. J Invest Dermatol. 2009;129:302-8.

14. Eidsmo L, Allan R, Caminschi I, van Rooijen N, Heath WR, Carbone FR. Differential migration of epidermal and dermal dendritic cells during skin infection. J Immunol. 2009;182:3165-72.

15. Guttman-Yassky E, Lowes MA, Fuentes-Duculan J, Whynot J, Novitskaya I, Cardinale I, et al. Major differences in inflammatory dendritic cells and their products distinguish atopic dermatitis from psoriasis. J Allergy Clin Immunol. 2007;119:1210-7.

16. Leung DY, Boguniewicz M, Howell MD, Nomura I, Hamid QA. New insights into atopic dermatitis. J Clin Invest. 2004;113:651-7.

17. Werfel T. The role of leukocytes, keratinocytes, and allergenspecific $\mathrm{IgE}$ in the development of atopic dermatitis. J Invest Dermatol. 2009;129:1878-91.

18. Elentner A, Finke D, Schmuth M, Chappaz S, Ebner S, Malissen $\mathrm{B}$, et al. Langerhans cells are critical in the development of atopic dermatitis-like inflammation and symptoms in mice. J Cell Mol Med. 2009;13:2658-72. 
19. Allam JP, Novak N. The pathophysiology of atopic eczema. Clin Exp Dermatol. 2006;31:89-93.

20. Jin H, He R, Oyoshi M, Geha RS. Animal models of atopic dermatitis. J Invest Dermatol. 2009;129:31-40.

21. Olivry T, Moore PF, Affolter VK, Naydan DK. Langerhans cell hyperplasia and IgE expression in canine atopic dermatitis. Arch Dermatol Res. 1996;288:579-85.

22. Bond E, Adams WC, Smed-Sorensen A, Sandgren KJ, Perbeck L, Hofmann A, et al. Techniques for time-efficient isolation of human skin dendritic cell subsets and assessment of their antigen uptake capacity. J Immunol Methods. 2009;348:42-56.

23. Marsella R, Girolomoni G. Canine models of atopic dermatitis: a useful tool with untapped potential. J Invest Dermatol. 2009; 129:2351-7.

24. Bice DE, Seagrave J, Green FH. Animal models of asthma: potential usefulness for studying health effects of inhaled particles. Inhal Toxicol. 2000;12:829-62.

25. Happ GM. Thyroiditis - a model canine autoimmune disease. Adv Vet Sci Comp Med. 1995;39:97-139.

26. Soon-Shiong P, Feldman E, Nelson R, Heintz R, Merideth N, Sandford $\mathrm{P}$, et al. Long-term reversal of diabetes in the large animal model by encapsulated islet transplantation. Transplant Proc. 1992;24:2946-7.

27. Day MJ. Antigen specificity in canine autoimmune haemolytic anaemia. Vet Immunol Immunopathol. 1999;69:215-24.

28. Ytterberg SR. Animal models of myopathy. Curr Opin Rheumatol. 1991;3:934-40.

29. Pascucci L, Mercati F, Gargiulo AM, Pedini V, Sorbolini S, Ceccarelli P. CD34 glycoprotein identifies putative stem cells located in the isthmic region of canine hair follicles. Vet Dermatol. 2006;17:244-51.

30. Nfon CK, Toka FN, Dawson H, Golde WT. characterization of Langerhans cells in porcine skin. Vet Immunol Immunopathol. 2008; $126: 236-47$.

31. von Bubnoff D, Koch S, Bieber T. Dendritic cells and atopic eczema/dermatitis syndrome. Curr Opin Allergy Clin Immunol. 2003;3:353-8.
32. Valladeau J, Saeland S. Cutaneous dendritic cells. Semin Immunol. 2005;17:273-83.

33. Marsella R, Olivry T, Nicklin C, Lopez J. Pilot investigation of a model for canine atopic dermatitis: environmental house dust mite challenge of high-IgE-producing beagles, mite hypersensitive dogs with atopic dermatitis and normal dogs. Vet Dermatol. 2006;17:24-35.

34. Olivry T, Naydan DK, Moore PF. Characterization of the cutaneous inflammatory infiltrate in canine atopic dermatitis. Am J Dermatopathol. 1997;19:477-86.

35. Olivry T, Deangelo KB, Dunston SM, Clarke KB, McCall CA. Patch testing of experimentally sensitized beagle dogs: development of a model for skin lesions of atopic dermatitis. Vet Dermatol. 2006;17:95-102.

36. Marsella R, Olivry T, Maeda S. Cellular and cytokine kinetics after epicutaneous allergen challenge (atopy patch testing) with house dust mites in high-IgE beagles. Vet Dermatol. 2006;17:11120.

37. Johnson-Huang LM, McNutt NS, Krueger JG, Lowes MA. Cytokine-producing dendritic cells in the pathogenesis of inflammatory skin diseases. J Clin Immunol. 2009;29:247-56.

38. Wollenberg A, Klein E. Current aspects of innate and adaptive immunity in atopic dermatitis. Clin Rev Allergy Immunol. 2007;33:35-44.

39. Wollenberg A, Wen S, Bieber T. Phenotyping of epidermal dendritic cells: clinical applications of a flow cytometric micromethod. Cytometry. 1999;37:147-55.

40. Ricklin Gutzwiller ME, Moulin HR, Zurbriggen A, Roosje P, Summerfield A. Comparative analysis of canine monocyte- and bone-marrow-derived dendritic cells. Vet Res. 2010;41:40.

41. Nestle FO, Di Meglio P, Qin JZ, Nickoloff BJ. Skin immune sentinels in health and disease. Nat Rev Immunol. 2009;9:67991.

42. Moore PF, Schrenzel MD, Affolter VK, Olivry T, Naydan D. Canine cutaneous histiocytoma is an epidermotropic Langerhans cell histiocytosis that expresses CD1 and specific beta 2-integrin molecules. Am J Pathol. 1996;148:1699-708. 